\title{
Introduction to Journal of Human-Robot Interaction Special Issue on Design
}

\author{
Lars Erik Holmquist \\ Grounded Innovation \\ and \\ Jodi Forlizzi \\ Human-Computer Interaction Institute and School of Design \\ Carnegie-Mellon University
}

Welcome to the Journal of Human-Robot Interaction's first special issue on design! We strongly believe that design is integral to the continued development of the field of HRI, and we have created this special issue to highlight the many ways in which the field of robotics research is influenced by design - and vice versa.

Much like computing has become increasingly mobile, ubiquitous, and part of everyday life, someday in the future we will find robots assisting humans in situations well beyond their current use. They may become assistants, workmates, entertainers, and even socializers. Robotic solutions are already increasingly applied to real-world problems such as our ageing society, renewable energy, climate control, emergency response, education, and exploration. Achieving a successful interplay between robots and humans will require careful consideration of the role and effect of robots in our lives. These societal problems require a holistic approach to the design and development of robots that meet human needs, address technical challenges, and foster acceptance in everyday settings, and this is one way in which design can help.

As robot technology becomes more commonplace, design aspects will become increasingly important. In this special issue, we explore the discipline of design as it supports holistic human-robot interaction. In essence, design is about understanding the current state and then designing an improved future state. The design community has established many methods for creating engaging artifacts and has branched out into subfields such as interaction design and product design, which are highly relevant for the HRI field. Designers have unique opportunities to improve the overall appeal and usefulness of robotic products well beyond their technical function and capabilities. Finally, designers are well-equipped to envision and understand the implications and potential of new technologies through techniques such as concept development, sketching, and lo-fi prototyping.

To highlight this rising importance of design in HRI, we have included a wide range of submissions in this publication. A unique feature of this special issue is that the authors of accepted papers are invited to present their paper at HRI 2014 (the 9th ACM/IEEE International Conference on Human-Robot Interaction, http://humanrobotinteraction.org/2014). This opportunity allows authors to present their work to a large audience and contribute toward improving the quality and profile of design papers at the HRI conference.

The interest from the research and design community was very strong for this special

\footnotetext{
Authors retain copyright and grant the Journal of Human-Robot Interaction right of first publication with the work simultaneously licensed under a Creative Commons Attribution License that allows others to share the work with an acknowledgement of the work's authorship and initial publication in this journal.
}

Journal of Human-Robot Interaction, Vol. 3, No. 1, 2014, Pages 1-3, DOI 10.5898/JHRI.3.1.Holmquist 
issue, and we were especially happy to receive submissions from a diverse set of authors; from established HRI researchers as well as artists, designers, and even special effects experts. In total, 19 papers were submitted in response to the call. All submissions that were suited to be a fit for the special issue were peer-reviewed by at least two external reviewers and the special issue editors. From these, we chose six papers for publication. Four of these are published as regular academic papers, presenting original research. Another two are published as essays, meaning that while the requirements for original research are lower, they present valuable insights into the history and development of design and HRI.

We open the issue with Oh and Park's essay, From Mechanical Metamorphosis to Empathic Interaction: A Historical Overview of Robotic Creatures. The authors give a broad historical overview of life-like and "intelligent" machines through the ages. This overview is by no means limited to robots in scientific research, but it also includes instances of robotic creatures in artistic and aesthetic contexts and a wealth of pointers to further study. We think this essay gives a useful yet refreshingly different view of the field, as it helps set the stage for the many different forms that robots have taken historically and will take in the future.

The following paper is Auger's Living With Robots: A Speculative Design Approach. The author addresses the field of domestic robots and the (so far) relative lack of success of such products. Framed in domestication theory and a family of artistically-influenced design methods, the paper presents a number of speculative design projects, stemming from various artists and designers as well as the author's own work. This methodical approach, while fairly well-known in the HCI community through the work of researchers and designers such as Bill Gaver, Anthony Dunne, and Fiona Raby, has had less impact on HRI to date. For the future success of robotic products, it will be important to consider a wide variety of approaches, and this paper presents some that can be radical as well as useful.

Next, we have Coactive Design: Designing Support for Interdependence in Joint Activity by Johnson, Bradshaw, Feltovich, Jonker, van Riemsdijk, and Sierhuis. The authors explore the question of what it means to have a robot as a teammate and describe an approach called coactive design to describe the interplay of humans and robots working together in a variety of domains. The "coactive" concept conveys the reciprocal and mutually constraining nature of actions and effects that are conditioned by coordination when robots and humans are working together. The paper offers a coactive system model and describes how it can be used for collaborative control of robotic systems, including a case study of the DARPA virtual robotics challenge, to show how the model can be applied. This is an important paper because it begins to tackle the messy research area of humans-in-the-loop with robotic systems.

In their paper, Designing Robots in the Wild: In Situ Prototype Evaluation for a Break Management Robot, Sabanovic, Reeder, and Kechavarzi share a set of in-situ studies of robotic artifacts. They deployed a variety of prototypes in office environments, e.g., on a person's desk, and used a selection of HCI methods to evaluate the different results. To deploy robots in real (or nearly real) settings continues to be very difficult due to the complicated technology that is usually involved, and this makes it hard to design studies that produce straightforward results. The main insights in this paper concern the overarching methodological issues raised by the different experiments, and this will be valuable as HRI research moves toward integrating robots in more realistic situations.

Hoffman and Ju's paper, Designing Robots With Movement in Mind, explores expressive movement and how it can benefit HRI design. They offer a new approach for doing so-pragmatic action - which is revolutionary for the HRI community, because it differs from a visual approach to designing motion that is standard. Quality of motion is critical for robots in lay contexts, as it is of critical importance in how non-expert users will react to and interact with robots. In the paper, the authors offer techniques for movement-centric design and illustrate these techniques through a series of well-detailed case studies. This paper is a rich archive for HRI and motion design and will help HRI researchers improve the ways in which robots are expressive to humans.

We close this special issue with our second essay, Sherer's Movie Magic Makes Better Social Robots: The Overlap of Special Effects and Character Robot Engineering. The author 
describes his collaboration with engineers and scientists to develop character robots, drawing from rich experience in working with animatronics-i.e., electronics and motor-driven puppetry-for film and haunted houses. He introduces the notion of character robots, and proposes that they can be divided into six types: work, entertainment, companion, security, killbot, and sex robots. The paper introduces the notion of a slave robot who does valued work without reward and forges a moral connection with an individual. The author also describes four traits of a character and shows how these can be applied to social robots, illustrating his concepts with a number of examples. This is a thought-provoking essay coming from an industry where mechanical creations already frequently interact with humans, containing much actionable information for the HRI community.

In closing, we want to thank all the authors and submitters for their interest and the great work they have shared with us! There were also many papers and projects that we, for various reasons, were not able to include this time around but that will certainly appear in JHRI or other venues at a later date. Overall it is clear that this kind of work is developing and maturing at an increasingly fast pace, which promises that great things are still ahead for the continued intermingling of HRI and design.

Authors' names and contact information: L. E. Holmquist, Grounded Innovation, 1134 Noe Street, San Francisco, CA 94114. Email: lars.erik.holmquist@gmail.com; J. Forlizzi, Human-Computer Interaction Institute and School of Design, Carnegie Mellon University, Pittsburgh, PN, USA. Email: forlizzi@cs.cmu.edu 